\title{
Properties of Cosmic Helium Isotopes Measured by the Alpha Magnetic Spectrometer
}

M. Aguilar, ${ }^{27}$ L. Ali Cavasonza, ${ }^{1}$ G. Ambrosi, ${ }^{33}$ L. Arruda, ${ }^{25}$ N. Attig, ${ }^{22}$ A. Bachlechner, ${ }^{1}$ F. Barao, ${ }^{25}$ A. Barrau, ${ }^{17}$ L. Barrin ${ }^{15}$ A. Bartoloni, ${ }^{38}$ S. Başeğmez-du Pree, ${ }^{18,{ }^{*}}$ R. Battiston,,${ }^{36,37}$ U. Becker, ${ }^{10}$ M. Behlmann, ${ }^{10}$ B. Beischer, ${ }^{1}$ J. Berdugo, ${ }^{27}$ B. Bertucci,${ }^{33,34}$ V. Bindi, ${ }^{20}$ W. de Boer ${ }^{23}$ K. Bollweg, ${ }^{21}$ B. Borgia, ${ }^{38,39}$ M. J. Boschini, ${ }^{29}$ M. Bourquin, ${ }^{16}$ E. F. Bueno, ${ }^{18}$ J. Burger, ${ }^{10}$ W. J. Burger, ${ }^{36}$ X. D. Cai, ${ }^{10}$ M. Capell, ${ }^{10}$ S. Caroff, ${ }^{3}$ J. Casaus, ${ }^{27}$ G. Castellini, ${ }^{14}$ F. Cervelli, ${ }^{35}$ Y. H. Chang, ${ }^{45}$ G. M. Chen, ${ }^{6,7}$ H. S. Chen, ${ }^{6,7}$ Y. Chen, ${ }^{16}$ L. Cheng, ${ }^{41}$ H. Y. Chou, ${ }^{45}$ V. Choutko, ${ }^{10}$ C. H. Chung, ${ }^{1}$ C. Clark,${ }^{10,21}$ G. Coignet, ${ }^{3}$ C. Consolandi, ${ }^{20}$ A. Contin, ${ }^{8,9}$ C. Corti, ${ }^{20}$ Z. Cui, ${ }^{41}$ K. Dadzie, ${ }^{10}$ Y. M. Dai, ${ }^{5}$ A. Datta, ${ }^{20}$ C. Delgado, ${ }^{27}$ S. Della Torre,${ }^{29}$ M. B. Demirköz,${ }^{2}$ L. Derome, ${ }^{17}$ S. Di Falco, ${ }^{35}$ V. Di Felice,${ }^{33, \dagger}$ C. Díaz, ${ }^{27}$ F. Dimiccoli, ${ }^{36}$ P. von Doetinchem, ${ }^{20}$ F. Dong, ${ }^{31}$ F. Donnini, ${ }^{33, \dagger}$ M. Duranti, ${ }^{33}$ A. Egorov, ${ }^{10}$ A. Eline, ${ }^{10}$ J. Feng, ${ }^{10}$ E. Fiandrini, ${ }^{33,34}$ P. Fisher, ${ }^{10}$ V. Formato, ${ }^{33, \dagger}$ Y. Galaktionov, ${ }^{10}$ C. Gámez, ${ }^{27}$ R. J. García-López, ${ }^{24}$ C. Gargiulo, ${ }^{15}$ H. Gast, ${ }^{1}$ I. Gebauer, ${ }^{23}$ M. Gervasi, ${ }^{29,30}$ F. Giovacchini, ${ }^{27}$ D. M. Gómez-Coral, ${ }^{28}$ J. Gong, ${ }^{31}$ C. Goy, ${ }^{3}$ V. Grabski, ${ }^{28}$ D. Grandi, ${ }^{29,30}$ M. Graziani, ${ }^{23}$ K. H. Guo, ${ }^{19}$ S. Haino, ${ }^{44}$ K. C. Han,${ }^{26}$ Z. H. He ${ }^{19}$ T. H. Hsieh,${ }^{10}$ H. Huang, ${ }^{44,}$ Z. C. Huang, ${ }^{19}$ M. Incagli, ${ }^{35}$ W. Y. Jang, ${ }^{13}$ Yi Jia, ${ }^{10}$ H. Jinchi, ${ }^{26}$ K. Kanishev,${ }^{36}$ B. Khiali, ${ }^{33, \dagger}$ G. N. Kim, ${ }^{13}$ Th. Kirn, ${ }^{1}$ M. Konyushikhin, ${ }^{10}$ O. Kounina, ${ }^{10}$ A. Kounine, ${ }^{10}$ V. Koutsenko, ${ }^{10}$ A. Kulemzin, ${ }^{10}$ G. La Vacca ${ }^{29,30}$ E. Laudi, ${ }^{15}$ G. Laurenti, ${ }^{8}$ I. Lazzizzera,${ }^{36,37}$ A. Lebedev, ${ }^{10}$ H. T. Lee, ${ }^{43}$ S. C. Lee, ${ }^{44}$ J. Q. Li, ${ }^{31}$ Q. Li, ${ }^{31}$ T. X. Li,${ }^{19}$ Z. H. Li, ${ }^{6}$ C. Light, ${ }^{20}$ C. H. Lin,${ }^{44}$ T. Lippert, ${ }^{22}$ Z. Liu, ${ }^{16}$ S. Q. Lu, ${ }^{19}$ Y. S. Lu, ${ }^{6}$ K. Luebelsmeyer, ${ }^{1}$ F. Luo, ${ }^{41}$ J. Z. Luo, ${ }^{31}$ Xi Luo, ${ }^{20}$ S. S. Lyu, ${ }^{19}$ F. Machate, ${ }^{1}$ C. Mañá, ${ }^{27}$ J. Marín, ${ }^{27}$ T. Martin, ${ }^{10,21}$ G. Martínez, ${ }^{27}$ N. Masi, ${ }^{8}$ D. Maurin, ${ }^{17}$ A. Menchaca-Rocha, ${ }^{28}$ Q. Meng,${ }^{31}$ D. C. Mo, ${ }^{19}$ M. Molero, ${ }^{27}$ P. Mott,,${ }^{10,21}$ L. Mussolin, ${ }^{33,34}$ T. Nelson, ${ }^{20}$ J. Q. Ni, ${ }^{19}$ N. Nikonov, ${ }^{1}$ F. Nozzoli, ${ }^{36}$ A. Oliva, ${ }^{27}$ M. Orcinha, ${ }^{25}$ M. Palermo, ${ }^{20}$ F. Palmonari, ${ }^{8,9}$ M. Paniccia, ${ }^{16}$ A. Pashnin, ${ }^{10}$ M. Pauluzzi, ${ }^{33,34}$ S. Pensotti, ${ }^{29,30}$ H. D. Phan, ${ }^{10}$ V. Plyaskin, ${ }^{10}$ V. Poireau, ${ }^{3}$ S. Poluianov, ${ }^{32}$ A. Popkow, ${ }^{20}$ X. M. Qi,${ }^{19}$ X. Qin, ${ }^{10}$ Z. Y. Qu, ${ }^{44}$ L. Quadrani, ${ }^{8,9}$ P. G. Rancoita, ${ }^{29}$ D. Rapin, ${ }^{16}$ A. Reina Conde, ${ }^{24}$

S. Rosier-Lees, ${ }^{3}$ A. Rozhkov, ${ }^{10}$ D. Rozza, ${ }^{29,30}$ R. Sagdeev, ${ }^{11}$ S. Schael, ${ }^{1}$ S. M. Schmidt, ${ }^{22}$ A. Schulz von Dratzig, ${ }^{1}$ G. Schwering, ${ }_{1}^{1}$ E. S. Seo, ${ }^{12}$ B. S. Shan, ${ }^{4}$ J. Y. Shi, ${ }^{31}$ T. Siedenburg, ${ }^{1}$ C. Solano, ${ }^{10}$ J. W. Song, ${ }^{41}$ Z. T. Sun,${ }^{6,7}$ M. Tacconi,${ }^{29,30}$ X. W. Tang, ${ }^{6}$ Z. C. Tang, ${ }^{6}$ J. Tian, ${ }^{33,34}$ Samuel C. C. Ting $\odot,{ }^{10,15}$ S. M. Ting, ${ }^{10}$ N. Tomassetti, ${ }^{33,34}$ J. Torsti, ${ }^{46}$ C. Tüysüzz, ${ }^{2}$ T. Urban, ${ }^{10,21}$ I. Usoskin, ${ }^{32}$ V. Vagelli, ${ }^{33,34,8}$ R. Vainio, ${ }^{46}$ E. Valente, ${ }^{38,39}$ E. Valtonen, ${ }^{46}$ M. Vázquez Acosta, ${ }^{24}$ M. Vecchi,${ }^{18,40}$ M. Velasco, ${ }^{27}$ J. P. Vialle, ${ }^{3}$ L. Q. Wang, ${ }^{41}$ N. H. Wang, ${ }^{41}$ Q. L. Wang, ${ }^{5}$ X. Wang, ${ }^{10}$ X. Q. Wang, ${ }^{6,7}$ Z. X. Wang, ${ }^{19}$ J. Wei ${ }^{16}$ Z. L. Weng, ${ }^{10}$ H. Wu, ${ }^{31}$ R. Q. Xiong, ${ }^{31}$ W. Xu, ${ }^{10,41}$ Q. Yan, ${ }^{10}$ Y. Yang, ${ }^{42}$ H. Yi, ${ }^{31}$ Y. J. Yu, ${ }^{5}$ Z. Q. Yu, ${ }^{6}$ M. Zannoni,${ }^{29,30}$ S. Zeissler, ${ }^{23}$ C. Zhang, ${ }^{6}$ F. Zhang, ${ }^{6}$ J. H. Zhang, ${ }^{31}$ Z. Zhang, ${ }_{10}^{10}$ F. Zhao, ${ }^{6,7}$ Z. M. Zheng, ${ }^{4}$ H. L. Zhuang, ${ }^{6}$ V. Zhukov, ${ }^{1}$ A. Zichichi, ${ }^{8,9}$ N. Zimmermann, ${ }^{1}$ and P. Zuccon ${ }^{36,37}$

(AMS Collaboration)

\footnotetext{
${ }^{1}$ I. Physics Institute and JARA-FAME, RWTH Aachen University, 52056 Aachen, Germany

${ }^{2}$ Department of Physics, Middle East Technical University (METU), 06800 Ankara, Turkey

${ }^{3}$ Université Grenoble Alpes, Université Savoie Mont Blanc, CNRS, LAPP-IN2P3, 74000 Annecy, France

${ }^{4}$ Beihang University (BUAA), Beijing 100191, China

${ }^{5}$ Institute of Electrical Engineering (IEE), Chinese Academy of Sciences, Beijing 100190, China

${ }^{6}$ Institute of High Energy Physics (IHEP), Chinese Academy of Sciences, Beijing 100049, China

${ }^{7}$ University of Chinese Academy of Sciences (UCAS), Beijing 100049, China

${ }^{8}$ INFN Sezione di Bologna, 40126 Bologna, Italy

${ }^{9}$ Università di Bologna, 40126 Bologna, Italy

${ }^{10}$ Massachusetts Institute of Technology (MIT), Cambridge, Massachusetts 02139, USA

${ }^{11}$ East-West Center for Space Science, University of Maryland, College Park, Maryland 20742, USA

${ }^{12}$ IPST, University of Maryland, College Park, Maryland 20742, USA

${ }^{13}$ CHEP, Kyungpook National University, 41566 Daegu, Korea

${ }^{14}$ CNR-IROE, 50125 Firenze, Italy

${ }^{15}$ European Organization for Nuclear Research (CERN), 1211 Geneva 23, Switzerland

${ }^{16}$ DPNC, Université de Genève, 1211 Genève 4, Switzerland

${ }^{17}$ Université Grenoble Alpes, CNRS, Grenoble INP, LPSC-IN2P3, 38000 Grenoble, France

${ }^{18}$ KVI-Center for Advanced Radiation Technology, University of Groningen, 9700 AB Groningen, The Netherlands

${ }^{19}$ Sun Yat-Sen University (SYSU), Guangzhou 510275, China

${ }^{20}$ Physics and Astronomy Department, University of Hawaii, Honolulu, Hawaii 96822, USA

${ }^{21}$ National Aeronautics and Space Administration Johnson Space Center (JSC), Houston, Texas 77058, USA
} 


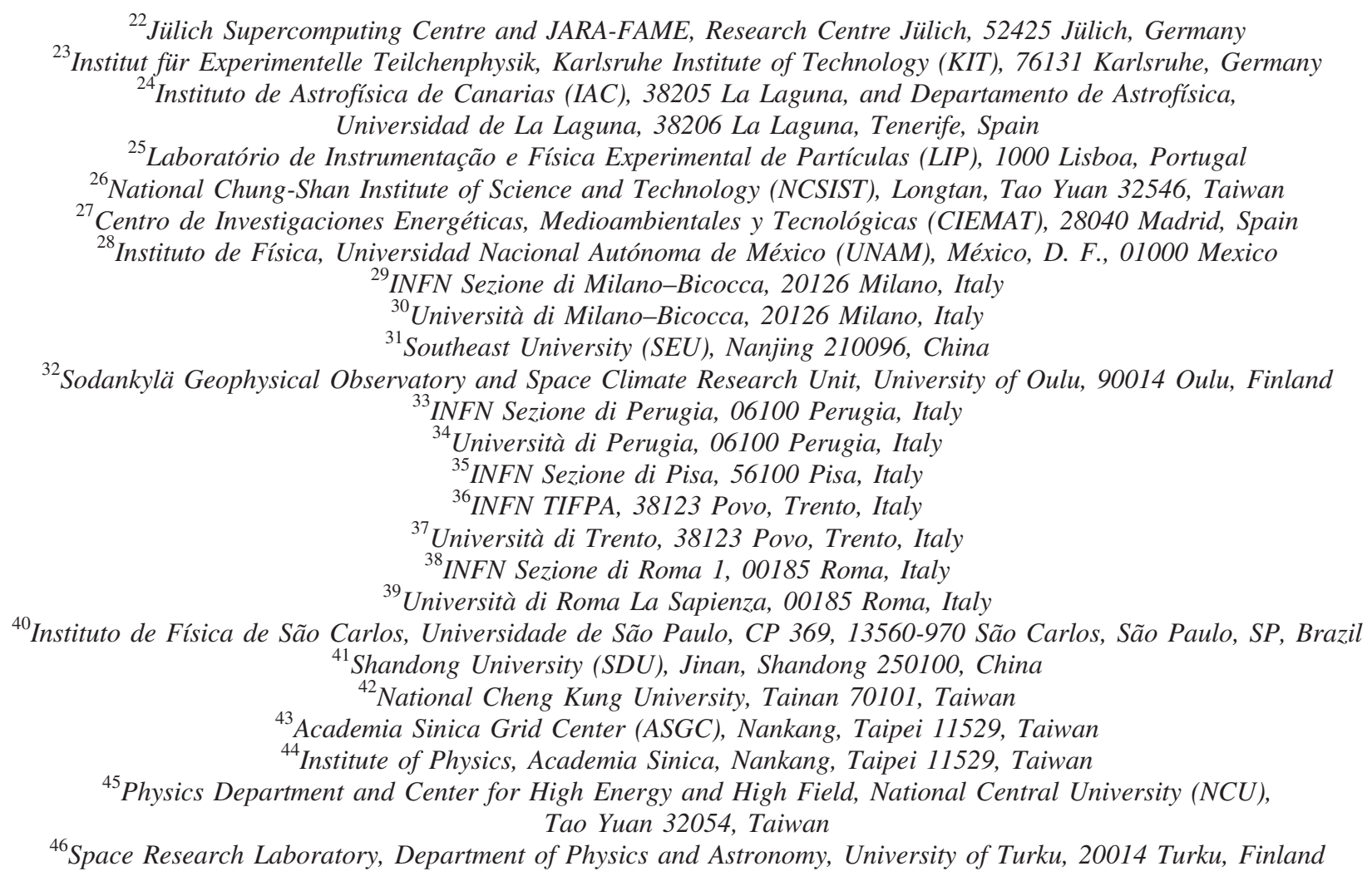

${ }^{22} J$ ülich Supercomputing Centre and JARA-FAME, Research Centre Jülich, 52425 Jülich, Germany

${ }^{23}$ Institut für Experimentelle Teilchenphysik, Karlsruhe Institute of Technology (KIT), 76131 Karlsruhe, Germany

${ }^{24}$ Instituto de Astrofísica de Canarias (IAC), 38205 La Laguna, and Departamento de Astrofísica, Universidad de La Laguna, 38206 La Laguna, Tenerife, Spain

${ }^{25}$ Laboratório de Instrumentação e Física Experimental de Partículas (LIP), 1000 Lisboa, Portugal ${ }^{26}$ National Chung-Shan Institute of Science and Technology (NCSIST), Longtan, Tao Yuan 32546, Taiwan

${ }^{27}$ Centro de Investigaciones Energéticas, Medioambientales y Tecnológicas (CIEMAT), 28040 Madrid, Spain

${ }^{28}$ Instituto de Física, Universidad Nacional Autónoma de México (UNAM), México, D. F., 01000 Mexico

${ }^{29}$ INFN Sezione di Milano-Bicocca, 20126 Milano, Italy

${ }^{30}$ Università di Milano-Bicocca, 20126 Milano, Italy

${ }^{31}$ Southeast University (SEU), Nanjing 210096, China

${ }^{32}$ Sodankylä Geophysical Observatory and Space Climate Research Unit, University of Oulu, 90014 Oulu, Finland

${ }^{33}$ INFN Sezione di Perugia, 06100 Perugia, Italy

${ }^{34}$ Università di Perugia, 06100 Perugia, Italy

${ }^{35}$ INFN Sezione di Pisa, 56100 Pisa, Italy

${ }^{36}$ INFN TIFPA, 38123 Povo, Trento, Italy

${ }^{37}$ Università di Trento, 38123 Povo, Trento, Italy

${ }^{38}$ INFN Sezione di Roma 1, 00185 Roma, Italy

${ }^{39}$ Università di Roma La Sapienza, 00185 Roma, Italy

${ }^{40}$ Instituto de Física de São Carlos, Universidade de São Paulo, CP 369, 13560-970 São Carlos, São Paulo, SP, Brazil

${ }^{41}$ Shandong University (SDU), Jinan, Shandong 250100, China

${ }^{42}$ National Cheng Kung University, Tainan 70101, Taiwan

${ }^{43}$ Academia Sinica Grid Center (ASGC), Nankang, Taipei 11529, Taiwan

${ }^{44}$ Institute of Physics, Academia Sinica, Nankang, Taipei 11529, Taiwan

${ }^{45}$ Physics Department and Center for High Energy and High Field, National Central University (NCU), Tao Yuan 32054, Taiwan

${ }^{46}$ Space Research Laboratory, Department of Physics and Astronomy, University of Turku, 20014 Turku, Finland

(Received 2 August 2019; revised manuscript received 2 October 2019; published 1 November 2019)

Precision measurements by the Alpha Magnetic Spectrometer (AMS) on the International Space Station of ${ }^{3} \mathrm{He}$ and ${ }^{4} \mathrm{He}$ fluxes are presented. The measurements are based on 100 million ${ }^{4} \mathrm{He}$ nuclei in the rigidity range from 2.1 to $21 \mathrm{GV}$ and 18 million ${ }^{3} \mathrm{He}$ from 1.9 to $15 \mathrm{GV}$ collected from May 2011 to November 2017. We observed that the ${ }^{3} \mathrm{He}$ and ${ }^{4} \mathrm{He}$ fluxes exhibit nearly identical variations with time. The relative magnitude of the variations decreases with increasing rigidity. The rigidity dependence of the ${ }^{3} \mathrm{He} /{ }^{4} \mathrm{He}$ flux ratio is measured for the first time. Below $4 \mathrm{GV}$, the ${ }^{3} \mathrm{He} /{ }^{4} \mathrm{He}$ flux ratio was found to have a significant long-term time dependence. Above $4 \mathrm{GV}$, the ${ }^{3} \mathrm{He} /{ }^{4} \mathrm{He}$ flux ratio was found to be time independent, and its rigidity dependence is well described by a single power law $\propto R^{\Delta}$ with $\Delta=-0.294 \pm 0.004$. Unexpectedly, this value is in agreement with the $\mathrm{B} / \mathrm{O}$ and $\mathrm{B} / \mathrm{C}$ spectral indices at high energies.

DOI: 10.1103/PhysRevLett.123.181102

Helium nuclei are the second most abundant cosmic ray. They consist of two isotopes, ${ }^{4} \mathrm{He}$ and ${ }^{3} \mathrm{He} .{ }^{4} \mathrm{He}$ are thought to be mainly produced and accelerated in astrophysical sources, while ${ }^{3} \mathrm{He}$ are overwhelmingly produced by the collisions of ${ }^{4} \mathrm{He}$ with the interstellar medium. The precise knowledge of the rigidity dependences of the helium isotope fluxes and their ratio provides important

Published by the American Physical Society under the terms of the Creative Commons Attribution 4.0 International license. Further distribution of this work must maintain attribution to the author(s) and the published article's title, journal citation, and DOI. information on the sources, acceleration, and propagation of cosmic rays [1].

Helium $\left({ }^{3} \mathrm{He},{ }^{4} \mathrm{He}\right)$ interaction cross sections with the interstellar medium $(p, \mathrm{He})$ are significantly smaller than those of heavier nuclei ( $\mathrm{Li}, \mathrm{Be}, \mathrm{B}, \mathrm{C}, \mathrm{N}, \mathrm{O}, \ldots)$. Therefore, helium travels larger distances, probing a larger Galactic volume. Explicitly, the ${ }^{3} \mathrm{He} /{ }^{4} \mathrm{He}$ ratio probes the properties of diffusion at larger distances [2].

${ }^{3} \mathrm{He}$ is produced by the fragmentation of ${ }^{4} \mathrm{He}$ with the interstellar medium, whereas $\mathrm{Li}, \mathrm{Be}$, and $\mathrm{B}$ are produced by multiple fragmentation channels of many heavy nuclei with the interstellar medium [3]. Therefore, the ${ }^{3} \mathrm{He} /{ }^{4} \mathrm{He}$ flux ratio provides unique input to cosmic-ray propagation models [4], distinctly different from the commonly studied 
secondary/primary ratios of heavier elements such as $\mathrm{B} / \mathrm{C}$ and $\mathrm{B} / \mathrm{O}[5-7]$.

Previously, the flux of helium nuclei has been measured by AMS based on 90 million events collected during the first $5 \mathrm{yr}$ of operation, revealing an unexpected hardening at high energies [8]. In addition, AMS reported the precise measurement of the electron, positron, proton, and helium fluxes as functions of time $[9,10]$.

The large acceptance and high precision of AMS allow the accurate measurements of temporal variation of ${ }^{3} \mathrm{He}$ and ${ }^{4} \mathrm{He}$ fluxes for the first time. Time correlations of different particle $\left(e^{+}, e^{-}, p,{ }^{3} \mathrm{He},{ }^{4} \mathrm{He}\right)$ flux variations are expected by models of cosmic-ray transport in the heliosphere [11]. The relative magnitude of these variations decreases with increasing rigidity. This is because the cosmic-ray transport in the heliosphere is rigidity dependent and related to changes in solar activity [12].

There are no previous measurements of the ${ }^{3} \mathrm{He} /{ }^{4} \mathrm{He}$ ratio as a function of rigidity. At lower energies and with larger errors, there have been measurements of helium isotope fluxes and their ratio as functions of the kinetic energy per nucleon [13].

In this Letter, precision measurements of the ${ }^{3} \mathrm{He}$ and ${ }^{4} \mathrm{He}$ fluxes and the ${ }^{3} \mathrm{He} /{ }^{4} \mathrm{He}$ flux ratio are presented from 1.9 to $15 \mathrm{GV}$ for ${ }^{3} \mathrm{He}$, from 2.1 to $21 \mathrm{GV}$ for ${ }^{4} \mathrm{He}$, and from 2.1 to $15 \mathrm{GV}$ for the ${ }^{3} \mathrm{He} /{ }^{4} \mathrm{He}$ flux ratio based on 100 million ${ }^{4} \mathrm{He}$ and 18 million ${ }^{3} \mathrm{He}$ nuclei collected by AMS from May 2011 to November 2017. The ${ }^{3} \mathrm{He}$ and ${ }^{4} \mathrm{He}$ fluxes are measured in 21 time periods of four Bartels rotations (108 days) each, ensuring enough statistics to measure ${ }^{3} \mathrm{He}$ to percent level accuracy. The total flux error at $10 \mathrm{GV}$ is $2.5 \%$ for ${ }^{3} \mathrm{He}$ and $2 \%$ for ${ }^{4} \mathrm{He}$.

The AMS detector description is presented in Ref. [14]. The elements used in this analysis are the magnet [15], the silicon tracker [16], the time of flight counters (TOF) [17], and the ring imaging Čerenkov detector (RICH) [18]. A summary of the detector performance and Monte Carlo (MC) simulation [19-22] is presented in Supplemental Material [23].

Selection.-In the first $6.5 \mathrm{yr}$ of operation, AMS has collected $1.07 \times 10^{11}$ cosmic-ray events. The collection time used in this analysis includes only those seconds during which the detector was in normal operating conditions and, in addition, the AMS was pointing within $40^{\circ}$ of the local zenith and the International Space Station (ISS) was outside of the South Atlantic Anomaly. Because of the influence of the geomagnetic field, the collection time for galactic cosmic rays increases with rigidity, reaching $1.31 \times 10^{8} \mathrm{~s}$ at $20 \mathrm{GV}$.

Helium events are required to be downward going and to have a reconstructed track in the inner tracker which passes through $L 1$ and a reconstructed velocity from the TOF $>0.3$. Track fitting quality criteria such as a $\chi^{2} /$ d.o.f. $<10$ in the bending coordinate are applied, similar to Ref. [8]. For events with a velocity above the Čerenkov threshold, we require additional quality criteria such as the number of hits in the Čerenkov ring $>4$, compatible velocity measurements in the TOF and RICH, i.e., $\left|1-\beta_{\mathrm{TOF}} / \beta_{\mathrm{RICH}}\right|<0.05$, and the charge measured in the $\mathrm{RICH}>1.2$.

To select only primary helium events $[8,24]$ with the same exposure time for both He isotopes, the measured velocity of the particles is required to be greater than that of ${ }^{3} \mathrm{He}$ with a rigidity equal to 1.2 times the maximum geomagnetic cutoff within the AMS field of view. The cutoff is calculated by backtracing particles [25] from the top of AMS out to 50 Earth radii using the most recent International Geomagnetic Reference Field [26].

Because of the multiple independent measurements of the charge, the selected helium sample contains a negligible background $\left(<10^{-3}\right)$ of particles with $Z>2$ at the top of the AMS [8]. The additional background in the ${ }^{3} \mathrm{He}$ sample is due to the fragmentation of ${ }^{4} \mathrm{He} \rightarrow{ }^{3} \mathrm{He}$ within the AMS materials, mostly carbon and aluminum [8]. To estimate this background, we used the reaction ${ }^{4} \mathrm{He}+(\mathrm{C}, \mathrm{Al}) \rightarrow{ }^{3} \mathrm{H}$, that has the same cross section as ${ }^{4} \mathrm{He}+(\mathrm{C}, \mathrm{Al}) \rightarrow{ }^{3} \mathrm{He}[4,27]$. This is illustrated in Fig. S1 in Supplemental Material [23], which shows the mass distribution of events interacting between $L 1$ and $L 2$ for data and $\mathrm{MC}$ simulation in the velocity range $0.9721<\beta<0.9954$. As seen, the peaks of protons, deuterons, and tritons are clearly observable, and the MC simulation agrees well with the data. The corresponding background of ${ }^{3} \mathrm{He}$ from ${ }^{4} \mathrm{He}$ interactions in the AMS material was estimated from the MC simulation to be less than $10 \%$ of the ${ }^{3} \mathrm{He}$ sample in the entire rigidity range, with uncertainties of $<1 \%$ for the ${ }^{3} \mathrm{He}$ flux.

To identify the He isotopes, a procedure based on the unfolding of the rigidity distribution [28] is used. After unfolding with the rigidity resolution function, the top of the instrument rigidity distribution of each isotope and the number of corresponding events are determined from velocity bins of width $\sim 0.2 \Delta \beta$. Examples of rigidity distributions and results of unfolding are presented in Fig. S2 in Supplemental Material [23] for velocity bins of the TOF, RICH-NaF, and RICH-Agl. Finally, the rate distribution $(\Gamma)$ in rigidity is obtained by dividing the number of events in each velocity bin by the corresponding velocity-dependent exposure time.

Analysis.-The fluxes of the helium isotopes are measured as functions of the rigidity in 26 bins from 1.92 to 21.1 GV chosen according to Ref. [8]. The isotropic flux $\Phi_{i}^{A}$ for an isotope of atomic mass number $A$ in the $i$ th rigidity bin $\left(R_{i}, R_{i}+\Delta R_{i}\right)$ is given by

$$
\Phi_{i}^{A}=\frac{\Gamma_{i}^{A}}{\mathcal{A}_{i}^{A} \epsilon_{i}^{A} \Delta R_{i}},
$$


where $\Gamma_{i}^{A}$ is the sum of the unfolded rates in rigidity bin $i$, $\mathcal{A}_{i}^{A}$ the effective acceptance, $\epsilon_{i}^{A}$ the trigger efficiency, and $\Delta R_{i}$ the bin width.

Extensive studies were made of the systematic errors. The systematic errors that affect the determination of $\Gamma_{i}^{A}$ include the uncertainties due to the resolution functions in the velocity and rigidity used in the unfolding procedure. The TOF velocity resolution function $\Delta(1 / \beta)$ has a Gaussian core of width 0.02 . The velocity resolution functions of the RICH-NaF and RICH-Agl are similar; the RICH-Agl has a pronounced Gaussian core characterized by a width of $7 \times 10^{-4}$ at $\beta=1$ and decreases with decreasing velocity to $6.3 \times 10^{-4}$ at $\beta \sim 0.953$. The velocity resolution functions of TOF and RICH are obtained from the MC simulation and validated at $\beta=1$ with data, as shown in Fig. S3 in Supplemental Material [23]. The rigidity resolution function, determined from the $\mathrm{MC}$ simulation, has been extensively verified with the data [8]. The systematic errors due to uncertainties in the rigidity resolution functions have been estimated by varying the width of the resolution function by $10 \%$. The systematic error from the determination of $\Gamma_{i}^{A}$ on the ${ }^{3} \mathrm{He}$ flux is $3 \%$ at $2 \mathrm{GV}$, is $1 \%$ from 3 to $10 \mathrm{GV}$, and increases up to $4.5 \%$ at $15 \mathrm{GV}$. For the ${ }^{4} \mathrm{He}$ flux, it is $4 \%$ below $3 \mathrm{GV}$, is $1 \%$ from 4 to $15 \mathrm{GV}$, and increases up to $3 \%$ at $21 \mathrm{GV}$.

Other sources of systematic error include uncertainties in the trigger efficiency, in the geomagnetic cutoff, and in the acceptance.

The trigger efficiency of ${ }^{3} \mathrm{He}$ and ${ }^{4} \mathrm{He}$ has been measured following a procedure similar to Ref. [8]. The systematic error on the fluxes associated with the trigger efficiency uncertainties is $<0.7 \%$ for ${ }^{3} \mathrm{He}$ and $<0.2 \%$ for ${ }^{4} \mathrm{He}$ over the entire rigidity range.

The geomagnetic cutoff factor was varied from 1.0 to 1.4 , resulting in a negligible systematic uncertainty $(<0.1 \%)$ in the whole rigidity range.

The effective acceptances $\mathcal{A}_{i}^{A}$ were calculated from the MC simulation and then corrected for differences between the data and simulated events related to (a) event reconstruction and selection, namely, in the efficiencies of track finding, charge determination, tracker quality cuts, and velocity quality cuts, and (b) inelastic interactions of helium nuclei in the AMS material. The total correction to the effective acceptances was found to be $<10 \%$ over the entire rigidity range. The systematic error on the ${ }^{3} \mathrm{He}$ and ${ }^{4} \mathrm{He}$ fluxes associated to (a) has been found by a comparison between the data and MC simulation to be $2.5 \%$ below $2 \mathrm{GV}$, to be $3.5 \%$ between 2 and $7 \mathrm{GV}$, and to decrease to $1.5 \%$ above $7 \mathrm{GV}$. The systematic error on (b) has been studied in detail in Ref. [8]. The corresponding systematic error on the ${ }^{3} \mathrm{He}$ flux was found to be $1.5 \%$ and, for ${ }^{4} \mathrm{He}$, $1 \%[22]$.

The variations of the trigger and reconstruction efficiencies were monitored as functions of time. A time-dependent systematic error due to the variations of trigger and reconstruction efficiencies for different time periods was estimated to be $1 \%$ in the whole rigidity range for both the ${ }^{3} \mathrm{He}$ and ${ }^{4} \mathrm{He}$ fluxes. All the other systematic errors discussed above are time independent.

Results.-The ${ }^{3} \mathrm{He}$ and ${ }^{4} \mathrm{He}$ fluxes are measured as functions of the rigidity from May 2011 to November 2017 in 21 time periods of four Bartels rotations (108 days) each. The fluxes and the flux ratio are presented in Tables I-XXI in Supplemental Material [23], including statistical and systematic errors. For the fluxes, the contributions of individual sources to the systematic error are added in quadrature to obtain the total systematic uncertainty. The sum of the two fluxes is consistent with Refs. [8,9] over the same time periods. For the ${ }^{3} \mathrm{He} /{ }^{4} \mathrm{He}$ flux ratio, the correlation of the systematic errors is taken into account to evaluate the total systematic error. Figure 1 shows the ${ }^{3} \mathrm{He}$ and ${ }^{4} \mathrm{He}$ fluxes as functions of time for five characteristic rigidity bins. As seen, both spectra exhibit

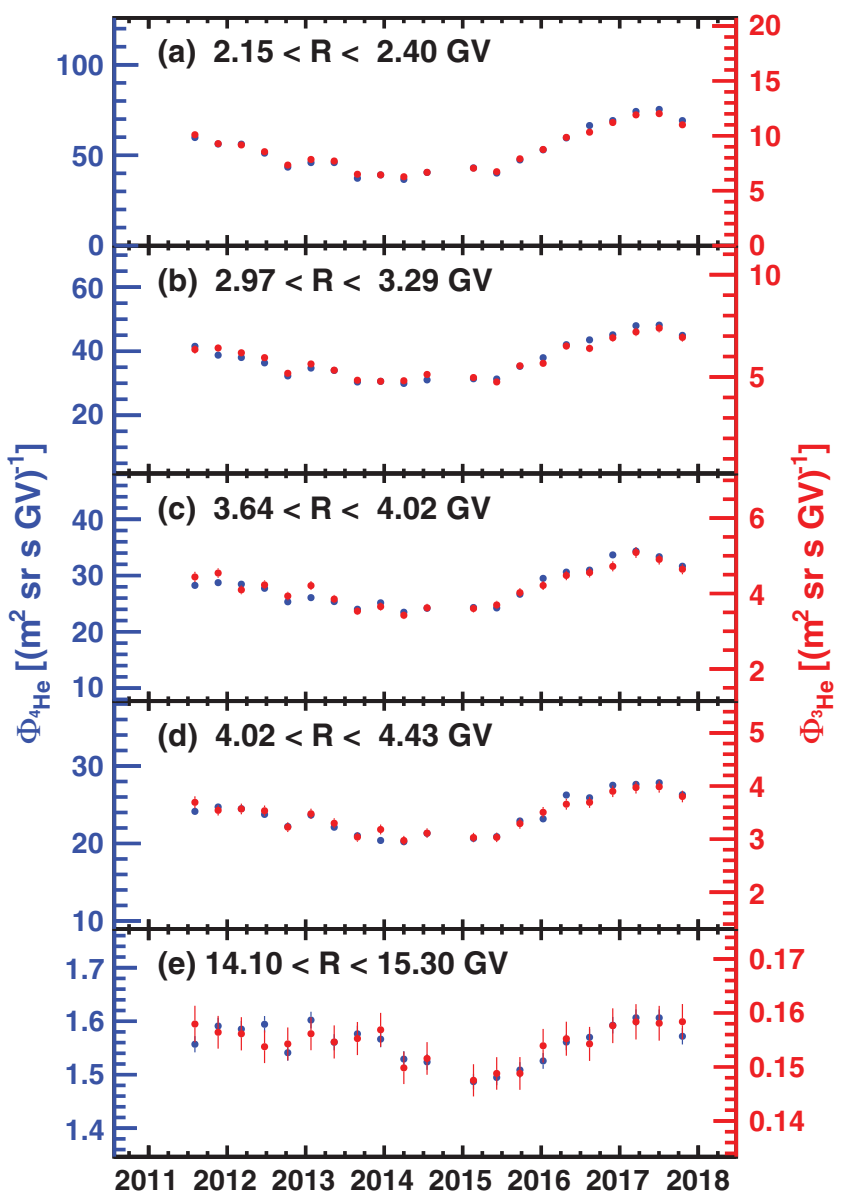

FIG. 1. The AMS ${ }^{3} \mathrm{He}$ (red points, right scale) and ${ }^{4} \mathrm{He}$ (blue points, left scale) fluxes as functions of time for five rigidity bins (a) from 2.15 to $2.40 \mathrm{GV}$, (b) from 2.97 to $3.29 \mathrm{GV}$, (c) from 3.64 to $4.02 \mathrm{GV}$, (d) from 4.02 to $4.43 \mathrm{GV}$, and (e) from 14.1 to $15.3 \mathrm{GV}$. The errors are the quadratic sum of the statistical and time-dependent systematic errors. 
nearly identical variations with time. The relative magnitude of the variations decreases with increasing rigidity; as seen in Fig. 1(a), $2.15<R<2.40 \mathrm{GV}$, the maximum and minimum are different by a factor of 2, whereas in Fig. 1(e), $14.1<R<15.3 \mathrm{GV}$, the difference is $10 \%$. The observed variations are compatible with those observed by AMS in the proton flux [9] and in the electron and positron fluxes [10]. Together, these provide important insights on the dynamics of solar modulation.

Figure 2 shows the ${ }^{3} \mathrm{He} /{ }^{4} \mathrm{He}$ flux ratio as a function of time for the same five rigidity bins. As seen, at low rigidities the ${ }^{3} \mathrm{He} /{ }^{4} \mathrm{He}$ flux ratio shows two distinct time behaviors similar to the $p / \mathrm{He}$ flux ratio in Fig. 4 in Ref. [9]. To characterize the time dependence of the ${ }^{3} \mathrm{He} /{ }^{4} \mathrm{He}$ flux ratio at low rigidities, a procedure similar to the one

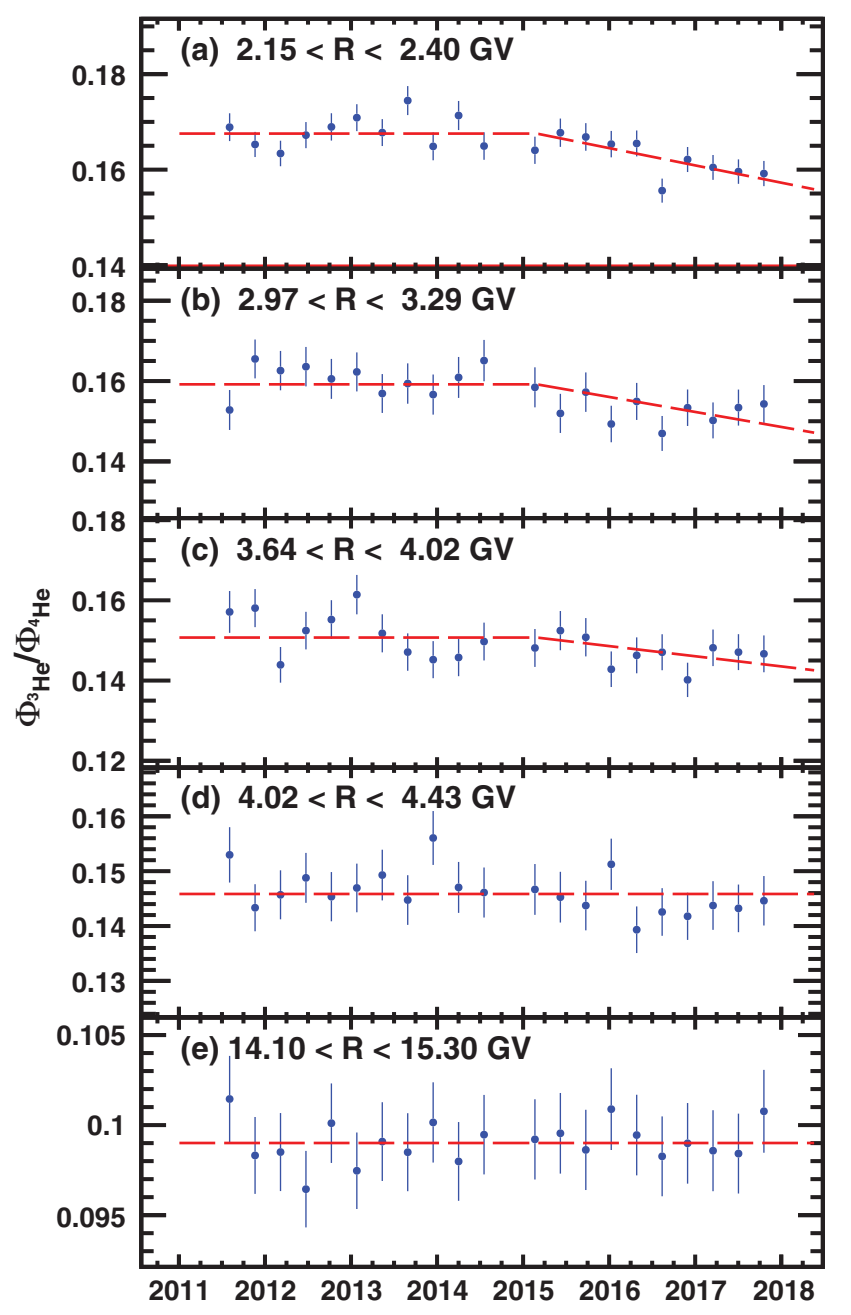

FIG. 2. The AMS ${ }^{3} \mathrm{He} /{ }^{4} \mathrm{He}$ flux ratio as a function of time for five rigidity bins (a) from 2.15 to $2.40 \mathrm{GV}$, (b) from 2.97 to $3.29 \mathrm{GV}$, (c) from 3.64 to $4.02 \mathrm{GV}$, (d) from 4.02 to $4.43 \mathrm{GV}$, and (e) from 14.1 to $15.3 \mathrm{GV}$. The errors are the quadratic sum of the statistical and time-dependent systematic errors. The dashed lines show fit results of Eq. (2) below $4 \mathrm{GV}$ and a constant fit above $4 \mathrm{GV}$. described in Ref. [9] was used. We performed fits of the flux ratio as a function of time for each rigidity bin according to

$$
r_{i}(t)= \begin{cases}a_{i} & t<\tilde{t} \\ a_{i}+b_{i}(t-\tilde{t}) & t \geq \tilde{t}\end{cases}
$$

where $r_{i}(t)$ is the ${ }^{3} \mathrm{He} /{ }^{4} \mathrm{He}$ flux ratio for rigidity bin $i$ as a function of time $t, a_{i}$ is the average ${ }^{3} \mathrm{He} /{ }^{4} \mathrm{He}$ flux ratio from May 2011 to $\tilde{t}$, and $b_{i}$ is the slope of the time variation after $\tilde{t}$. Below $4 \mathrm{GV}$, the fits yield a $\tilde{t}$ compatible with the average transition time found in the $p /$ He flux ratio of (February 28, 2015) \pm 42 days [9]. The results of the fits are also shown (dashed red lines) in Fig. 2 for $\tilde{t}$ fixed to February 28, 2015 [9]. As seen, the ${ }^{3} \mathrm{He} /{ }^{4} \mathrm{He}$ flux ratio exhibits a long-term time variation at low rigidities that gradually decreases with increasing rigidities. Above $4 \mathrm{GV}$, the ratio is found to be consistent with a constant value at the $95 \%$ C.L.

The measured ${ }^{3} \mathrm{He}$ and ${ }^{4} \mathrm{He}$ time-averaged fluxes and the corresponding ${ }^{3} \mathrm{He} /{ }^{4} \mathrm{He}$ flux ratio for the $6.5 \mathrm{yr}$ period are reported in Table XXII in Supplemental Material [23] as a function of the rigidity including statistical and systematic errors. Figure S4 in Supplemental Material [23] shows the time-averaged ${ }^{3} \mathrm{He}$ and ${ }^{4} \mathrm{He}$ fluxes together with the range of time variation.

To compare with previous experiments [13], the ${ }^{3} \mathrm{He}$ and ${ }^{4} \mathrm{He}$ fluxes and their ratio have also been measured using the same unfolding procedure described above from 0.5 to $10 \mathrm{GeV} / n$ as functions of the kinetic energy per nucleon, $E_{K}=M(\gamma-1) / A$, where $\gamma=1 / \sqrt{1-\beta^{2}}$ and $M$ and $A$ are the ${ }^{3} \mathrm{He}$ or ${ }^{4} \mathrm{He}$ mass and atomic mass number, respectively. The time-averaged ${ }^{3} \mathrm{He}$ and ${ }^{4} \mathrm{He}$ fluxes and

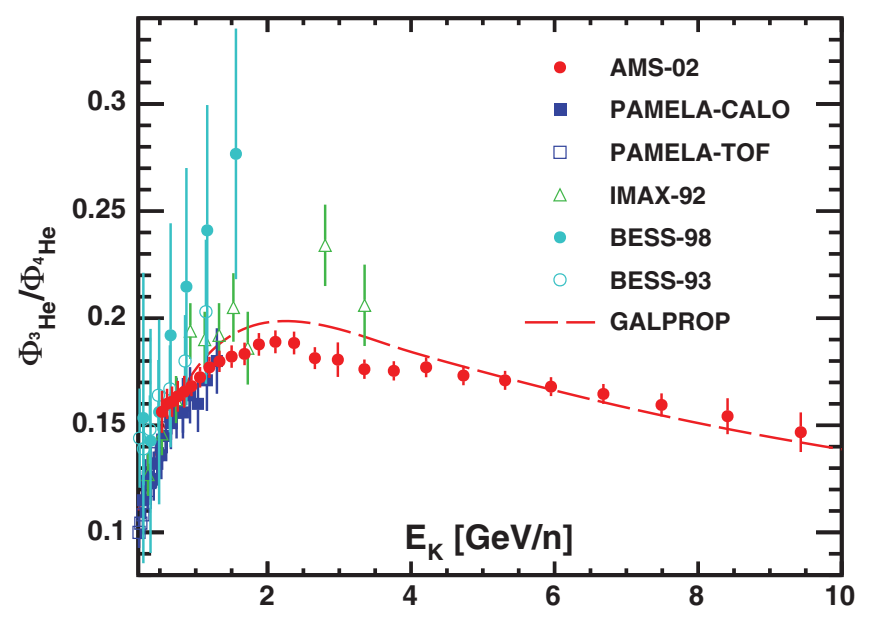

FIG. 3. The AMS ${ }^{3} \mathrm{He} /{ }^{4} \mathrm{He}$ ratio with total errors as a function of the kinetic energy per nucleon, together with earlier experiments [13]. The dashed line shows the prediction of the GALPROP model [29] with the average solar modulation, in the force-field approximation [30], corresponding to the data collection period. 
their ratio, including statistical and systematic errors, are reported in Table XXIII in Supplemental Material [23] as functions of $E_{K}$. The ${ }^{3} \mathrm{He} /{ }^{4} \mathrm{He}$ ratio as a function of the kinetic energy per nucleon is shown in Fig. 3 together with earlier experiments and the GALPROP model [29] with the average solar modulation, in the force-field approximation [30] corresponding to the data collection period. The AMS result provides a precision measurement and covers a large energy range.
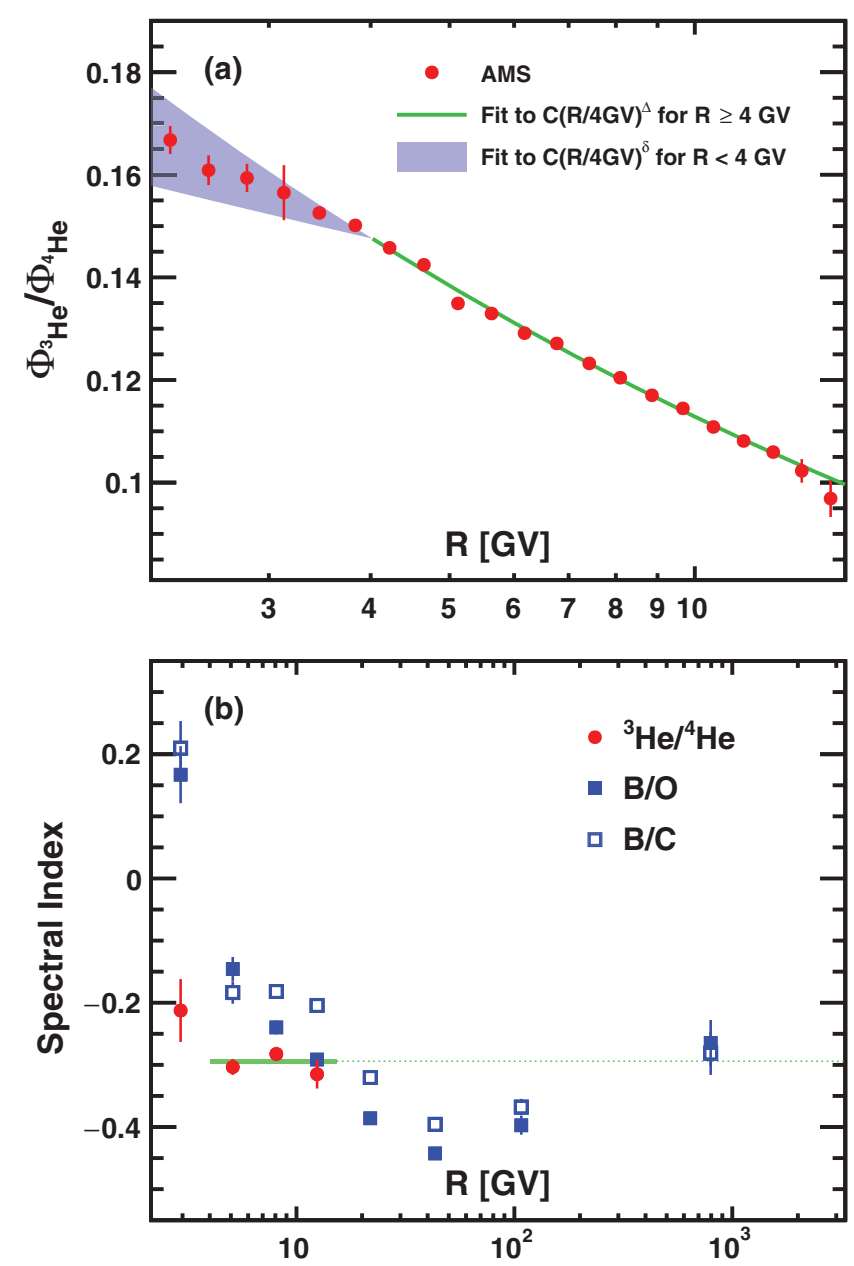

FIG. 4. (a) The time-averaged ${ }^{3} \mathrm{He} /{ }^{4} \mathrm{He}$ flux ratio as a function of rigidity $R$ (red points) with statistical and uncorrelated systematic errors added in quadrature. The solid green curve shows a single power law fit $C(R / 4 \mathrm{GV})^{\Delta}$ above $4 \mathrm{GV}$. The shaded blue area indicates the results of single power law fits to $C(R / 4 \mathrm{GV})^{\delta}$ below $4 \mathrm{GV}$ for each of the 21 time periods. (b) The ${ }^{3} \mathrm{He} /{ }^{4} \mathrm{He}$ flux ratio spectral index (red points) as a function of $R$. As seen, above $4 \mathrm{GV}$, the spectral index shows no dependence with rigidity (green band). The extrapolation of $\Delta$ to higher energies is shown (green dotted line). The B/O (blue filled squares) and $\mathrm{B} / \mathrm{C}$ (blue open squares) flux ratio spectral indices $[5,6]$ are also shown. As seen, the ${ }^{3} \mathrm{He} /{ }^{4} \mathrm{He}$ spectral index extrapolated to the highest energies is in good agreement with the $\mathrm{B} / \mathrm{O}$ and $\mathrm{B} / \mathrm{C}$ spectral indices.
The time-averaged ${ }^{3} \mathrm{He} /{ }^{4} \mathrm{He}$ flux ratio as a function of rigidity is shown in Fig. 4(a). Above $4 \mathrm{GV}$, the ${ }^{3} \mathrm{He} /{ }^{4} \mathrm{He}$ flux ratio is time independent and is well described with a single power law, $C(R / 4 \mathrm{GV})^{\Delta}$. A fit to the data yields $\Delta=-0.294 \pm 0.004$ and $C=0.1476 \pm 0.0004$, with $\chi^{2} /$ d.o.f. $=15.7 / 13$. Below $4 \mathrm{GV}$, to study the time and rigidity dependence of the ${ }^{3} \mathrm{He} /{ }^{4} \mathrm{He}$ flux ratio, fits to $C(R / 4 \mathrm{GV})^{\delta}$ for each period of four Bartels rotations were performed. The results of the fits are shown as a shaded band in Fig. 4(a) and yield an average $\delta$ of $\langle\delta\rangle=$ $-0.21 \pm 0.02$ with a time variation of \pm 0.05 . Different from the $\mathrm{B} / \mathrm{O}$ and $\mathrm{B} / \mathrm{C}$ flux ratios, which show a maximum around 4 GV [see Figs. S9(c) and S10(c) in Ref. [6]], the ${ }^{3} \mathrm{He} /{ }^{4} \mathrm{He}$ flux ratio is steadily decreasing with the rigidity. Because of different propagation volumes and different inelastic interaction cross sections of $\mathrm{He}, \mathrm{B}, \mathrm{C}$, and $\mathrm{O}$, the $\mathrm{B} / \mathrm{O}$ and $\mathrm{B} / \mathrm{C}$ flux ratio rigidity dependence may be different from the ${ }^{3} \mathrm{He} /{ }^{4} \mathrm{He}$ flux ratio rigidity dependence, and this provides important information to discriminate among different cosmic-ray models $[1,2,4]$. The comparison of our data with models will be presented in a forthcoming publication [31].

Figure 4(b) shows the ${ }^{3} \mathrm{He} /{ }^{4} \mathrm{He}$ flux ratio spectral index as a function of $R$ for four independent rigidity bins. As seen, above $4 \mathrm{GV}$, the spectral index shows no dependence with rigidity with an average value of $\Delta=-0.294 \pm 0.004$. The extrapolation of $\Delta$ to higher energies is shown as a dotted line. The $\mathrm{B} / \mathrm{O}$ and $\mathrm{B} / \mathrm{C}$ flux ratio spectral indices $[5,6]$ are shown as blue filled squares and blue open squares, respectively. As seen, the ${ }^{3} \mathrm{He} /{ }^{4} \mathrm{He}$ spectral index extrapolation to the highest energies is in agreement with the $\mathrm{B} / \mathrm{O}$ and $\mathrm{B} / \mathrm{C}$ spectral indices.

In conclusion, precision measurements of the cosmic-ray ${ }^{3} \mathrm{He}$ and ${ }^{4} \mathrm{He}$ isotope fluxes and their ratio are presented as functions of rigidity from 1.9 to $15 \mathrm{GV}$ for ${ }^{3} \mathrm{He}$, from 2.1 to $21 \mathrm{GV}$ for ${ }^{4} \mathrm{He}$, and from 2.1 to $15 \mathrm{GV}$ for the ${ }^{3} \mathrm{He} /{ }^{4} \mathrm{He}$ flux ratio based on 100 million ${ }^{4} \mathrm{He}$ and 18 million ${ }^{3} \mathrm{He}$ nuclei collected by AMS from May 2011 to November 2017. Below $4 \mathrm{GV}$, the ${ }^{3} \mathrm{He} /{ }^{4} \mathrm{He}$ flux ratio shows a significant long-term time dependence. Different from the B/C and $\mathrm{B} / \mathrm{O}$ flux ratios, which show a maximum around $4 \mathrm{GV}$, the ${ }^{3} \mathrm{He} /{ }^{4} \mathrm{He}$ flux ratio was found to be decreasing with rigidity; below $4 \mathrm{GV}$, the ratio is well described by a single power law $\propto R^{\delta}$ with $\langle\delta\rangle=-0.21 \pm 0.02$ and a time variation of \pm 0.05 . Above $4 \mathrm{GV}$, the ${ }^{3} \mathrm{He} /{ }^{4} \mathrm{He}$ flux ratio was found to be time independent, and its rigidity dependence is well described by a single power law $\propto R^{\Delta}$ with $\Delta=-0.294 \pm 0.004$. Unexpectedly, this value is in good agreement with the $\mathrm{B} / \mathrm{O}$ and $\mathrm{B} / \mathrm{C}$ spectral indices at high energies.

We are grateful for important physics discussions with Pasquale Blasi, Fiorenza Donato, Jonathan Ellis, Jonathan Feng, Mischa Malkov, Igor Moskalenko, and Subir Sarkar. We thank former NASA Administrator Daniel S. Goldin for 
his dedication to the legacy of the ISS as a scientific laboratory and his decision for NASA to fly AMS as a DOE payload. We also acknowledge the continuous support of the NASA leadership, particularly William $\mathrm{H}$. Gerstenmaier, and of the JSC and MSFC flight control teams that have allowed AMS to operate optimally on the ISS for over eight years. We are grateful for the support of Jim Siegrist and his staff of the DOE including resources from the National Energy Research Scientific Computing Center under Contract No. DE-AC02-05CH11231. We also acknowledge the continuous support from MIT and its School of Science, Michael Sipser, and Boleslaw Wyslouch. Research supported by São Paulo Research Foundation (FAPESP) Grant No. 2014/19149-7, Brazil; CAS, NSFC, MOST, the provincial governments of Shandong, Jiangsu, Guangdong, and the China Scholarship Council, China; the Academy of Finland (Center of Excellence ReSoLVE), Project No. 307411, Finland; CNRS/IN2P3, CNES, Enigmass, and the ANR, France; Pascale Ehrenfreund, DLR under Grant No. 50001403 and Jülich Aachen Research Alliance - High Performance Computing under Project No. JARA0052, Germany; INFN and ASI under ASIINFN Agreements No. 2013-002-R.0 and No. 2014-037-R.0, Italy; Center for High Energy Physics and NRF under Grant No. NRF-2018R1A6A1A06024970 at Kyungpook National University, Korea; the Consejo Nacional de Ciencia y Tecnología and UNAM, Mexico; NWO under Grant. No. 680-1-004, The Netherlands; FCT under Grant No. PTDC/FIS/122567/2010, Portugal; CIEMAT, Instituto de Astrofísica de Canarias, CDTI, and SEIDI-MINECO under Grants No. ESP2017-87055-C2-1-P, No. SEV-20150548, No. MDM-2015-0509, and No. RyC-2013-14660, Spain; the Swiss National Science Foundation (SNSF), federal and cantonal authorities, Switzerland; Academia Sinica and the Ministry of Science and Technology (MOST) under Grants No. 103-2112-M-006-018-MY3, No. 105-2112-M-001-003, and No. CDA-105-M06, former Presidents of Academia Sinica Yuan-Tseh Lee and Chi-Huey Wong and former Ministers of MOST Maw-Kuen Wu and Luo-Chuan Lee, Taiwan; the Turkish Atomic Energy Authority under Grants No. 2017TAEK(CERN)A5.H6.F215, Turkey; and NSF Grants No. 14255202 and No. 1551980 , Wyle Laboratories Grant No. 2014/T72497, and NASA NESSF Grant No. HELIO15F-0005, USA. We gratefully acknowledge the strong support from CERN including Fabiola Gianotti, and the CERN IT department including Bernd Panzer-Steindel, and from the European Space Agency including Johann-Dietrich Wörner and Simonetta Di Pippo.

*Also at Nikhef, 1098 XG Amsterdam, The Netherlands. ${ }^{\dagger}$ Also at ASI Space Science Data Center (SSDC), 00133 Roma, Italy.

${ }^{\ddagger}$ Also at Wuhan University, Wuhan 430072, China.

${ }^{\S}$ Present address: ASI, I-00133 Roma, Italy.
[1] I. A. Grenier, J. H. Black, and A. W. Strong, Annu. Rev. Astron. Astrophys. 53, 199 (2015); P. Blasi, Astron. Astrophys. Rev. 21, 70 (2013); A. W. Strong, I. V. Moskalenko, and V. S. Ptuskin, Annu. Rev. Nucl. Part. Sci. 57, 285 (2007).

[2] G. Jóhannesson et al., Astrophys. J. 824, 16 (2016).

[3] Y. Génolini, D. Maurin, I. V. Moskalenko, and M. Unger, Phys. Rev. C 98, 034611 (2018).

[4] B. Coste, L. Derome, D. Maurin, and A. Putze, Astron. Astrophys. 539, A88 (2012); N. Tomassetti, Astrophys. Space Sci. 342, 131 (2012).

[5] M. Aguilar et al., Phys. Rev. Lett. 117, 231102 (2016).

[6] M. Aguilar et al., Phys. Rev. Lett. 120, 021101 (2018).

[7] C. D. Orth, A. Buffington, G. F. Smoot, and T. S. Mast, Astrophys. J. 226, 1147 (1978); R. Dwyer and P. Meyer, Astrophys. J. 322, 981 (1987); M. Simon, H. Spiegelhauer, W. K. H. Schmidt, F. Siohan, J. F. Ormes, V. K. Balasubrahmanyan, and J. F. Arens, Astrophys. J. 239, 712 (1980); J. J. Engelmann et al., Astron. Astrophys. 233, 96 (1990); W. R. Webber et al., in Proceedings of 19th International Cosmic Ray Conference, La Jolla (1985), Vol. 2, p. 16; D. Muller, S. P. Swordy, P. Meyer, J. L'Heureux, and J. M. Grunsfeld, Astrophys. J. 374, 356 (1991); J. Buckley, J. Dwyer, D. Mueller, S. Swordy, and K. K. Tang, Astrophys. J. 429, 736 (1994); M. Aguilar et al., Astrophys. J. 724, 329 (2010); A. D. Panov et al., in Proceedings of the 23rd International Cosmic Ray Conference Mérida, edited by R. Caballero, J. C. D’Olivo, G. Medina-Tanco, L. Nellen, F. A. Sánchez, and J.F. Valdés-Galicia (Universidad Nacional Autónoma de México, Mexico City, 2008), Vol. 2, pp. 3-6; H. S. Ahn et al., Astropart. Phys. 30, 133 (2008); A. Obermeier, P. Boyle, J. Hörandel, and D. Müller, Astrophys. J. 752, 69 (2012); O. Adriani et al., Astrophys. J. 791, 93 (2014).

[8] M. Aguilar et al., Phys. Rev. Lett. 115, 211101 (2015); 119, 251101 (2017).

[9] M. Aguilar et al., Phys. Rev. Lett. 121, 051101 (2018).

[10] M. Aguilar et al., Phys. Rev. Lett. 121, 051102 (2018).

[11] E. E. Vos and M. S. Potgieter, Astrophys. J. 815, 119 (2015); I. Cholis, D. Hooper, and T. Linden, Phys. Rev. D 93, 043016 (2016); C. Corti, V. Bindi, C. Consolandi, and K. Whitman, Astrophys. J. 829, 8 (2016); J. Gieseler, B. Heber, and K. Herbst, J. Geophys. Res. Space Phys. 122, 10964 (2017); N. Tomassetti, M. Orcinha, F. Barão, and B. Bertucci, Astrophys. J. Lett. 849, L32 (2017); M. J. Boschini, S. Della Torre, M. Gervasi, G. La Vacca, and P. G. Rancoita, Adv. Space Res. 62, 2859 (2018).

[12] M. S. Potgieter, Living Rev. Solar Phys. 10, 3 (2013).

[13] O. Reimer et al., Astrophys. J. 496, 490 (1998); J. Z. Wang et al., Astrophys. J. 564, 244 (2002); Z. D. Myers et al., in Proceedings of the 28th International Cosmic Rays Conference (ICRC 2003), 1805, Tokyo, Japan (Universal Academy Press, Inc., Tokyo, 2003); E. Mocchiutti (WIZARD/Caprice Collaboration), in Proceedings of the 28th International Cosmic Rays Conference (ICRC 2003), 1809, Tokyo, Japan (Universal Academy Press, Inc., Tokyo, 2003); O. Adriani et al., Astrophys. J. 818, 68 (2016).

[14] A. Kounine, Int. J. Mod. Phys. E 21, 1230005 (2012); S. Rosier-Lees, in Proceedings of the Astroparticle Physics TEVPA/IDM, Amsterdam, 2014 (unpublished); S. Ting, Nucl. Phys. B, Proc. Suppl. 243-244, 12 (2013); S.-C. 
Lee, in Proceedings of the 20th International Conference on Supersymmetry and Unification of Fundamental Interactions (SUSY 2012), Beijing, 2012 (unpublished); M. Aguilar, in Proceedings of the XL International Meeting on Fundamental Physics, Centro de Ciencias de Benasque Pedro Pascual, 2012 (unpublished); S. Schael, in Proceedings of the 10th Symposium on Sources and Detection of Dark Matter and Dark Energy in the Universe, Los Angeles, 2012 (unpublished); B. Bertucci, Proc. Sci. EPS-HEP2011 (2011) 67; M. Incagli, AIP Conf. Proc. 1223, 43 (2010); R. Battiston, Nucl. Instrum. Methods Phys. Res., Sect. A 588, 227 (2008).

[15] K. Lübelsmeyer et al., Nucl. Instrum. Methods Phys. Res., Sect. A 654, 639 (2011).

[16] B. Alpat et al., Nucl. Instrum. Methods Phys. Res., Sect. A 613, 207 (2010).

[17] V. Bindi et al., Nucl. Instrum. Methods Phys. Res., Sect. A 743, 22 (2014).

[18] M. Aguilar et al., Nucl. Instrum. Methods Phys. Res., Sect. A 614, 237 (2010); F. Giovacchini (on behalf of AMSRICH Collaboration), Nucl. Instrum. Methods Phys. Res., Sect. A 766, 57 (2014).

[19] J. Allison et al., IEEE Trans. Nucl. Sci. 53, 270 (2006); Nucl. Instrum. Methods Phys. Res., Sect. A 835, 186 (2016); S. Agostinelli et al., Nucl. Instrum. Methods Phys. Res., Sect. A 506, 250 (2003).

[20] A. Boudard, J. Cugnon, J.-C. David, S. Leray, and D. Mancusi, Phys. Rev. C 87, 014606 (2013); S. Leray, D. Mancusi, P. Kaitaniemi, J. C. David, A. Boudard, B.
Braunn, and J. Cugnon, J. Phys. Conf. Ser. 420, 012065 (2013).

[21] J. Ranft, Phys. Rev. D 51, 64 (1995).

[22] I. Tanihata et al., Phys. Lett. 160B, 380 (1985).

[23] See Supplemental Material at http://link.aps.org/ supplemental/10.1103/PhysRevLett.123.181102 for supporting figures and tables.

[24] J. Alcaraz et al., Phys. Lett. B 494, 193 (2000).

[25] J. Alcaraz et al., Phys. Lett. B 484, 10 (2000).

[26] C. C. Finlay et al., Geophys. J. Int. 183, 1216 (2010); E. Thébault et al., Earth Planets Space 67, 79 (2015).

[27] W. R. Webber, AIP Conf. Proc. 203, 294 (1990); A. Kh. Abdurakhimov et al., Nucl. Phys. A362, 376 (1981).

[28] M. Behlmann, P. Zuccon, C. Delgado, and F. Giovacchini, Proc. Sci. ICRC2017 (2017) 162.

[29] We used Galprop WebRun; A. E. Vladimirov, S. W. Digel, G. Jóhannesson, P. F. Michelson, I. V. Moskalenko, P. L. Nolan, E. Orlando, T. A. Porter, and A. W. Strong, Comput. Phys. Commun. 182, 1156 (2011); with parametrization from R. Trotta, G. Jóhannesson, I. V. Moskalenko, T. A. Porter, R. Ruiz de Austri, and A. W. Strong, Astrophys. J. 729, 106 (2011).

[30] L. J. Gleeson and W. I. Axford, Astrophys. J. 154, 1011 (1968).

[31] AMS Collaboration, Physics Reports, The Alpha Magnetic Spectrometer (AMS) on the International Space Station: Part II-Results from the First Seven Years. 